Williams Smith, H. \& Crabb, W. E. (1956). J. gen. Microbiol. 15, 556-574

\title{
The Typing of Escherichia coli by Bacteriophage: its Application in the Study of the E. coli Population of the Intestinal Tract of Healthy Calves and of Galves Suffering from White Scours
}

\author{
By H. WILLIAMS SMITH AND W. E. CRABb
}

Animal Health Trust, Lilystone Hall, Stock, Essex

\begin{abstract}
SUMMARY: A bacteriophage method of classifying strains of Escherichia coli which inhabit the alimentary tract of cattle has been evolved. It was possible to divide the strains into a large number of types by this method. Varying proportions of strains isolated from the faeces of human beings, sheep, pigs and poultry were also typable by the phages employed. This method of phage typing was used to study the origin and behaviour of the $E$. coli population in the alimentary tract of healthy cows and calves, and of calves suffering from white scours, in one self-contained herd (herd A) and to a lesser extent in twenty-seven other herds. Several types of $E$. coli were often found in the same faecal specimen in cases of scouring, as well as in healthy calves. Seventy different phage types were found in the faeces of the healthy calves in herd A, many occurring infrequently. Thirty-two different types were discovered in the faeces of the scouring calves in this herd; only one strain of one type was found in scouring calves that was not found also in healthy calves. Two types were found commonly in the calves but rarely in the cows. The examination of strains of $E$. coli isolated from faeces of cows and calves at daily and weekly intervals indicated that some types usually remained dominant for a week or so and were then gradually succeeded in dominance by other types. In some animals frequent and sudden changes were apparent; in others one type might be the only type isolated from the faeces for a number of weeks continuously. Changes of dominant phage-type commonly occurred in faeces of calves during the time they were suffering from white scours. The mother did not appear to be a frequent source from which a calf acquired its $E$. coli; the calf pens themselves seemed a more probable source. Exceedingly large numbers of $E$. coli were found in the faeces of healthy and scouring calves during the first 14 days of life, a period when white scours occurs; very much smaller numbers were found in older animals. Studies on material from the twentyseven other herds supported the findings in herd A; many phage types of $E$. coli were found in these herds that had not been found in herd $A$. The aetiology of calf scours is discussed in the light of these and other observations.
\end{abstract}

Escherichia coli is well recognized as having an important association with serious intestinal disorders of infants, calves and possibly the young of other species of animals. It is not intended to review the literature on the association of $E$. coli with white scours in calves since this has been ably carried out by Lovell (1955). It is noteworthy, however, that considerable advances have been made recently in the study of this disease, particularly by Lovell and his colleagues. These workers have elucidated the mechanism of the protective action of colostrum and have shown that the $K$ antigens of $E$. coli and their antibodies are associated with protection from the clinical disease. Attention was also paid to the epidemiology of the disease (see also Wood, 1955). The 
calves used in these investigations by Lovell and colleagues were obtained and brought to one centre before they had consumed any colostrum. Most of them were then given colostrum deprived of one or more of its natural components. Consequently, we decided to study the epidemiology of the disease under normal farm conditions. The present paper deals with such a study on a selfcontained commercial herd (A), special attention being paid to the origin and behaviour of the $E$. coli population of the alimentary tract of healthy calves and cows, as well as of calves suffering from white scours. Less intensive examinations were also conducted on calves from twenty-seven other herds.

We decided to evolve a bacteriophage method for classifying the strains of Escherichia coli we isolated from calves because it represented a new approach to a problem on which all the available epidemiological evidence is of a serological nature, and because it has been successfully employed in the study of several diseases caused by other bacteria. Earlier work (Nicolle, LeMinor, Buttiaux \& Ducrest, 1952), too, had shown that $E$. coli from human beings could be classified by means of phages.

\section{METHODS}

\section{Phage typing}

The isolation of phages. A search for suitable phages was first made by modifications of the cross-culture technique (Fisk, 1942). The results were not satisfactory and the search was continued by using the more classical method of examining specimens of sewage and faeces. Such specimens were incubated in Difco broth at various temperatures between 20 and $37^{\circ}$ for 6-24 hr., sometimes with the addition of cultures of Escherichia coli hitherto regarded as untypable. They were then centrifuged at 3000 r.p.m. for $45 \mathrm{~min}$. and the supernatant fluids either heated to $58^{\circ}$ for $30 \mathrm{~min}$. or treated with chloroform (Fredericq, 1950). These fluids were spotted on to plates of nutrient agar previously spread with different broth cultures of strains of $E$. coli. The plates were incubated at $28^{\circ}$ for $18 \mathrm{hr}$. Phage action was revealed by zones of complete or semicomplete lysis, or by discrete plaques. Activity due to colicines only was confirmed by inability to transmit; such preparations were discarded.

Purification and propagation of phages. Lytic areas were picked from the plates referred to above and mixed, in different dilutions, with a broth culture of the susceptible strain of Escherichia coli, spread over a plate of nutrient agar and incubated at $28^{\circ}$. A discrete plaque was picked as soon as plaques were distinctly visible and replated with the propagating strain. This process was repeated twice. Finally, a discrete plaque with some of the surrounding bacterial growth was picked into broth and incubated at $28^{\circ}$ until lysis occurred. More susceptible bacterial culture was then added and the process repeated until a high-titre phage preparation was judged to have been obtained. Any bacteria present were then killed by heating to $58^{\circ}$ for $30 \mathrm{~min}$. Occasionally this procedure also destroyed the phage content, so preparations of heatsensitive phage were sterilized of bacteria by shaking with a few drops of chloroform (Fredericq, 1950). 
Titration of phage preparations. Tenfold dilutions of the phage preparations were made in phosphate buffer $\left(\mathrm{KH}_{2} \mathrm{PO}_{4}, 3.4 \mathrm{~g}\right.$.; $\mathrm{Na}_{2} \mathrm{HPO}_{4}, 6 \mathrm{~g}$.; distilled water, $1000 \mathrm{ml}$.) A broth culture of the susceptible strain of Escherichia coli was spread evenly over the surface of an agar plate, allowed to dry, and then spotted with one drop $\left(\frac{1}{150} \mathrm{ml}\right.$.) of each dilution of the phage preparation. Plates were incubated at $28^{\circ}$ for $18 \mathrm{hr}$. and then read. The highest dilution which produced a large number of plaques that were semi-confluent or nearly so was chosen as the critical test dilution, i.e. that suitable for routine typing tests. Although it is customary in most methods of phage typing to select the highest dilution which produces confluent lysis as the critical test dilution, it was deliberately decided not to do this in the present studies since a few of the preparations inevitably contained colicines as well as phage particles; the critical test dilution adopted prevented any resulting confusion.

Method of setting-up tests. A modification of the method devised by Wilson \& Atkinson (1945) for the phage typing of Staphylococcus aureus was employed. Nutrient agar plates were dried at $37^{\circ}$ for $2 \mathrm{hr}$. with their lids partly open. Four drops ( $0.08 \mathrm{ml}$.) of an $18 \mathrm{hr}$. broth culture of the strain of Escherichia coli to be typed were spread evenly over the surface of one of the nutrient agar plates by means of a glass spreader. When these had dried, the phage preparations in their critical test dilutions were spotted on to each plate by means of a dropping pipette (150 drops/ml.). For the sake of convenience, the bottom of each plate was marked with a grease pencil into a number of squares equal to the number of phage preparations in use. When the drops had been absorbed the plates were incubated at $28^{\circ}$ for $18 \mathrm{hr}$. and read. Many phage preparations were discarded because tests carried out with a selection of strains of $E$. coli showed that they had an identical action. Finally, a group of 16 different phages, A, B, C, D, E, G, H, L, M, O, T, X, $Z_{1}, Z_{2}, Z_{3}$ and $Z_{4}$, were chosen for routine use in typing strains of bovine origin. Another 8 phages, $Z_{5}-Z_{12}$, were developed for typing strains isolated from other species of animals.

In preliminary studies, with a rich nutrient agar medium and incubation at $37^{\circ}$, some of the phages even in high concentrations occasionally exhibited no lytic action on some of the strains of Escherichia coli which were known to be susceptible to them. Attempts were made to overcome this by using different media and by using the cultures diluted; the latter had the undesirable effect of enhancing colicine action. The present method, particularly with the incubation temperature of $\mathbf{2 8}^{\circ}$, was much more satisfactory. Many strains were retyped by this method several times during a period of $1 \frac{1}{2}$ years and, apart from the variations inherent in any method of phage typing that depends on the pattern reactions given by strains with a number of test phages, they showed no significant variation in their susceptibility.

The isolation and identification of strains of Escherichia coli. Deep rectal swabs were taken and cultured on to plates of MacConkey agar which were incubated at $37^{\circ}$ for $24 \mathrm{hr}$. A number of colonies, usually 10, from each plate were subcultured separately into tubes of nutrient broth and of MacConkey broth. The latter were incubated at $44^{\circ}$ in a water bath for $24 \mathrm{hr}$. Cultures which produced acid and gas in this medium were considered to be Escherichia coli 
faecal type 1 and the corresponding cultures in nutrient broth, which in the meantime had been incubating at $37^{\circ}$, were immediately available for phage typing; the remaining cultures were discarded.

Nutrient agar. The nutrient agar used was prepared by solidifying Difco Nutrient Broth no. B3 with $\mathbf{1 . 5} \%$ (w/v) New Zealand agar.

\section{Epidemiological studies}

Herd $A$. 'This dairy herd, in which autumn and winter calving was practised, consisted of approximately 65 Shorthorn cows and their followers. It was self-contained, no cows or calves having been purchased in the last 6 years, and all the cows were machine-milked. The indoor accommodation consisted of one long byre at the end of which were ten pens, separated from each other by low wooden partitions, in which all the calves spent their first 3-4 months of life. The number of calves kept in each pen was mainly determined by their total number. When a cow was observed to be in the early stages of labour she was moved to an empty pen where she remained until $24 \mathrm{hr}$. after the calf was born. The calf remained in that pen for a few days only and was transferred to another with other calves of approximately the same age. All calves were given their mothers' colostrum, usually by bucket, for the first 4 days. They were fed bulk milk twice daily for the next month, when the feeding with milk substitutes began.

White scours had occurred in the calves in this herd for the last few years and all cases had responded to treatment with streptomycin. It was agreed that, should any cases occur during the present investigation, streptomycin therapy should be withheld until the last possible moment compatible with saving the calf's life, and that in the meantime the diet of milk should be replaced by one of milk and water or water only, in the hope that this would render the disease less acute and provide us with a longer period during which observations could be made. From time to time it was possible to introduce some variations in the procedures in this herd. They will be referred to when necessary.

Between August 1954 and April 1955, 60 calves were born and 16 of them developed white scours. Four of 25 calves born between August and November, 4 of 23 calves born between November and January and 8 of 12 calves born between January and April developed white scours. Much less information was available about the other herds studied.

\section{RESULTS}

The distribution of different phage types of Escherichia coli in cows and calves in herd $A$ and other herds

The main results of phage typing 1365 cultures from 63 healthy cows, 2500 cultures from 36 healthy calves and 622 cultures from 16 scouring calves in herd $A$ and 237 cultures from 49 healthy calves and 531 cultures from 82 scouring calves in 27 other herds are shown in Table 1. The types listed are only those that formed not less than $1 \%$ of the total number of cultures examined from at 
least one of the five sources. Phages $Z_{3}$ and $Z_{4}$ are not included in Table 1 since none of the cultures upon which they were active belonged to this category. The cultures from herd A were obtained from August 1954 to April 1955. During this time the faeces of some of the calves and cows were examined every day for several days and others were only examined once. Consequently, the frequency distribution of the different phage types may not be accurate. The cultures from the other herds were obtained between February and July 1955. None of these calves was examined more than once.

Herd $\boldsymbol{A}$. The total number of different phage types found in the faeces of the healthy cows, healthy calves and scouring calves in herd A was 69, 70 and 32 respectively. Since many of the types were found only very infrequently, it is probable that the comparatively smaller number of cultures examined from scouring calves accounted for the smaller number of types found in them. Only 22 types from the cows contained $1.0 \%$ or more of the total number of strains examined from this source and, including the untypable strains, these types together formed $82.7 \%$ of the whole. The corresponding figures for the healthy calves and scouring calves in herd A were 22 and $87.0 \%$ and 20 and $97 \cdot 3 \%$ respectively. Of all the cultures examined from scouring calves, it is noteworthy that only one culture was of a type not also found in cultures from the faeces of healthy calves. Only $18 \%$ of the cultures from the latter belonged to types that were not also found in the scouring calves. Most of the phage types present in the calves were found also in the cows and vice versa, although there were some variations between the frequency distribution of the phage types in the two groups that were probably significant. Phage type 2, for example, the most common type isolated from the faeces of healthy and scouring calves forming 16.0 and $16.7 \%$ of the whole respectively, comprised only $0.7 \%$ of the strains from cows; this type was also quite uncommon in the faeces of calves from the other herds. Again, phage type 22 comprised $8.1 \%$ of the strains from healthy calves in herd $A$ but was never isolated from the cows. These types, however, were rather exceptional.

Other herds. The number of phage types found in the faeces of the healthy calves and scouring calves in these herds was 46 and 65 respectively. There were 21 types found in the healthy calves that were not found in the scouring calves and 38 types found in the latter that were not found in the former. The proportion of cultures from scouring calves which belonged to types found also in healthy calves was $53 \%$; the corresponding figure for healthy calves was $63 \%$. These differences between scouring and healthy calves were much greater than those which existed in herd $A$. This was not surprising since the cultures came from 27 herds in all, only a relatively small number originating from each herd, these sometimes having been isolated from scouring calves only or healthy calves only. No one type found in the scouring calves from these herds comprised more than $7.5 \%$ of all the cultures examined, indicating that, taking the herds as a whole, no predominant phage type was associated with scours. Many different phage types were found in the calves in these herds that had not been found in herd A. For example, as far as scouring calves were concerned, $\mathbf{4 9} \%$ of the cultures examined from these herds belonged to 50 phage types 
which had not been found in herd A; and $\mathbf{3 0} \%$ of the cultures from herd $\mathbf{A}$ belonged to 17 types not found in these herds. A much higher proportion of cultures from these herds was untypable than was the case with those from herd $A$, a fact that was not surprising since the phages had been developed for use in this herd.

\section{The phage typing of strains of Escherichia coli from other animals}

A small number of strains of Escherichia coli from animals other than cattle was phage typed to determine whether the method might be of any use in studying the distribution of $\boldsymbol{E}$. coli in these animals.

Human beings. Of 50 strains isolated from the faeces of 50 people, only 17 were typable by means of the 16 phages used for typing the bovine strains. A further 5 strains were typed by the use of another 8 phages, $Z_{5}-Z_{12}$. Seven of the types had also been found in the faeces of cattle. Not more than 5 strains belonged to the same phage types.

Pigs. Of 50 strains isolated from the faeces of 50 pigs maintained on farm A, 23 were lysed by one or more of the phages of the 'bovine' group; of these, 13 belonged to types which had been found in either the cows or the calves. A further 10 were typable by means of the $Z_{5}-Z_{12}$ phages, Not more than 3 strains belonged to any one type.

A similar number of cultures from pigs kept on $c .50$ different farms were examined. Of these, 30 were acted upon by phages of the 'bovine' group, 22 of which belonged to types which had been found in the farm $A$ cattle. $A$ further 6 strains were typable by means of the phages $Z_{5}-Z_{12}$. No one type comprised more than 3 of the 50 strains.

Sheep. All except 6 of 50 strains isolated from the faeces of 50 sheep, few of which came from the same flock, were lysed by the bovine' group of phages; thirty-eight belonged to types which had been found in cattle. A further two strains were acted upon by the additional phages. No one type contained more than 4 strains.

Poultry. Of 50 strains of Escherichia coli isolated from the faeces of 50 chickens that belonged to different flocks, 35 were phage-typed by the 'bovine' group of phages. Of these, 22 belonged to types that had been found in cattle. A further 10 strains were typable by the $Z_{5}-Z_{12}$ group. No more than 3 strains fell into one type.

\section{The distribution of different phage-types amongst 150 cultures of Escherichia coli isolated from the same faecal specimen}

The number of different phage types of Escherichia coli found by examining 150 colonies from single specimens of faeces obtained from 10 different healthy calves or cows belonging to herd A was studied. The same loopful of faeces was cultured on 4 plates of MacConkey agar to ensure that 150 well-separated colonies could be picked in all. The number of types present in each specimen varied from one to eight, the exact grouping of the cultures in each specimen according to phage type being $150 ; 149,1 ; 101,49 ; 146,3,1 ; 125,21,2,2$; 

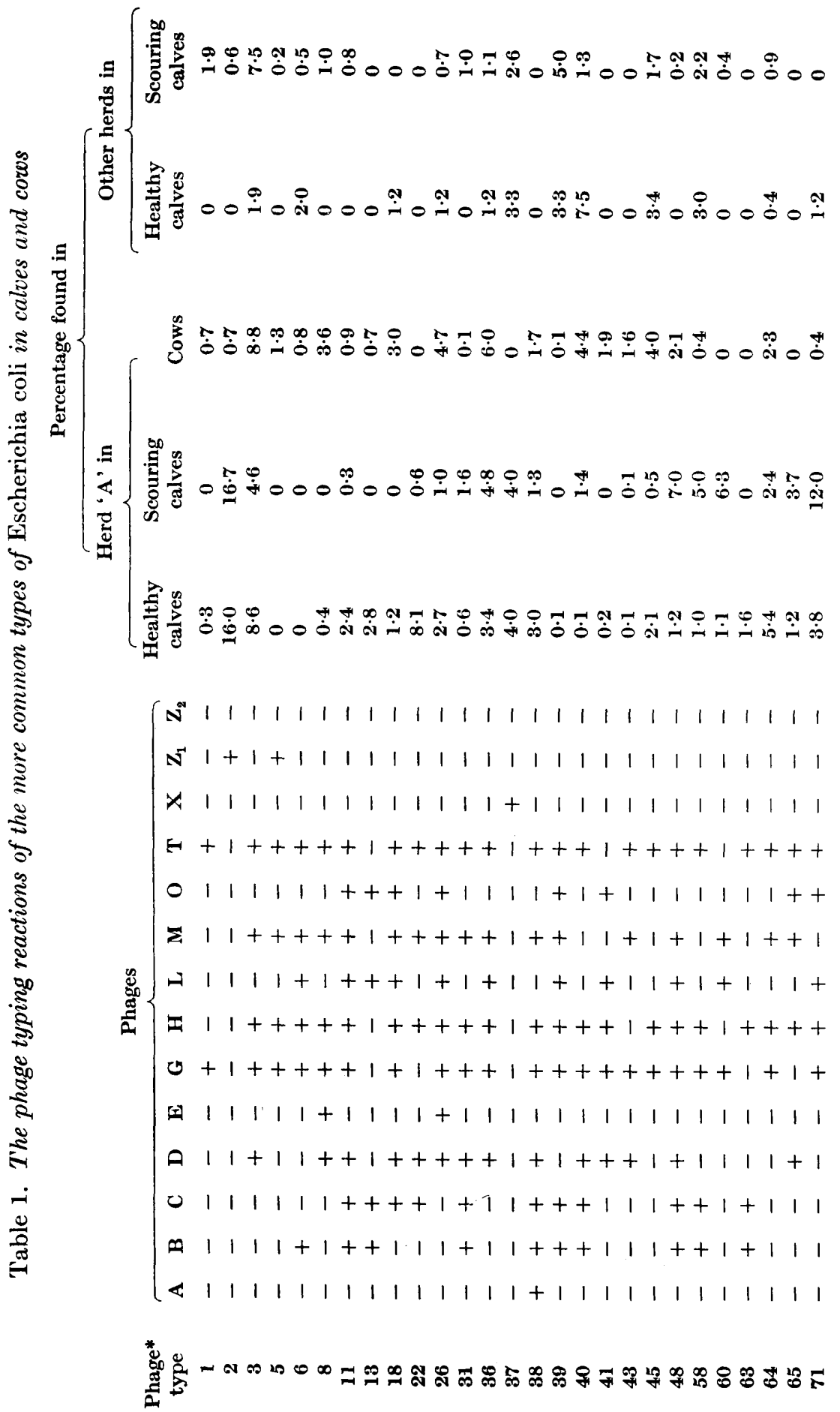


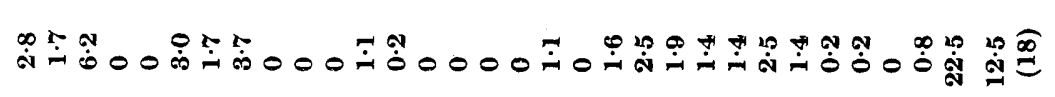

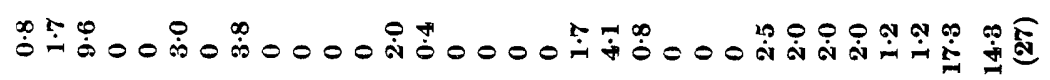

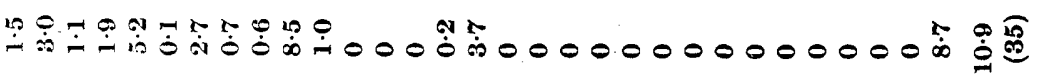

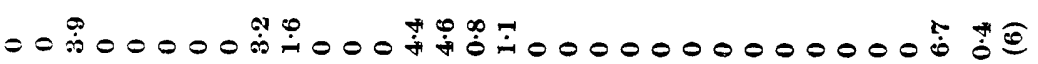

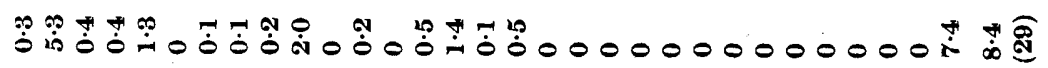

$\begin{array}{lllllllllllllllllllllllllllllll}1 & 1 & 1 & 1 & 1 & 1 & 1 & 1 & 1 & 1 & 1 & 1 & 1 & 1 & 1 & 1 & 1 & 1 & 1 & 1 & 1 & + & 1 & 1 & 1 & 1 & 1 & 1 & 1 & 1 & 1\end{array}$

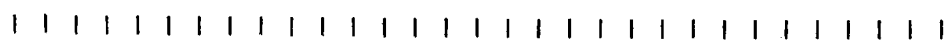

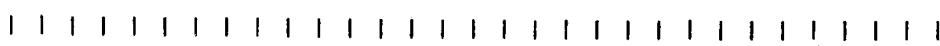
$++11++111+t++111+t+111+t+t++1+1$ $|1| 1+1 \mid 1++11+1++11+111++1111111$ $1+11+1|1|+t++1|1|++1|1|+11+1+1$

$111++1+1++1++1+111+111++1111111$ $++11++1+1+t+1+1+1++11+1+t++1+1$ $++|1+| 1|+t+1++| 1|+| 1+t+t+11++11$

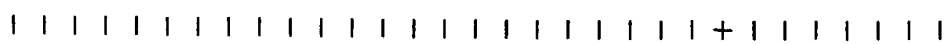

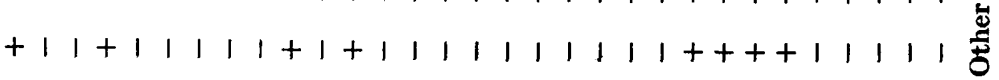
$1+t|1| 1|1| 1+1111111+1111111+1111$ $1++11111111+11$ i $11+t+11+1+1++1+1$

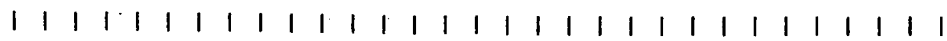

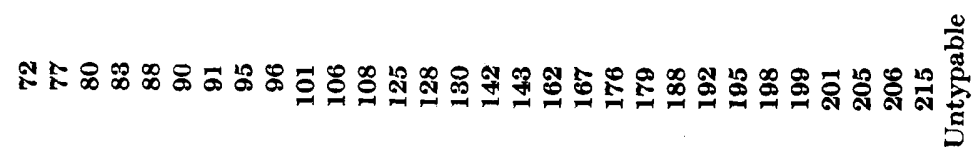


$89,38,13,6,4 ; 86,45,11,5,3 ; 60,60,27,1,1,1$; and $120,10,10,3,3,2,1,1$. As will be seen later, when 10 or even fewer colonies were examined from each specimen of faeces it was usual to find at least 2 types to be present. This multiplicity of types existing in the same animal was by no means found to be confined to the bovine species. The number of types found amongst 10 colonies from each of 10 faecal specimens from pigs varied from 2 to 8 , the average being 5.3. The corresponding figures for sheep were 1 to 6 and $2 \cdot 8$, for chickens 1 to 2 and $1 \cdot 6$ and for human beings 1 to 7 and $2 \cdot 8$.

The phage types of Escherichia coll present in the faeces of 25 cows in herd $A$ at each of six consecutive weekly examinations

Four colonies of Escherichia coli picked from MacConkey plates inoculated with individual faeces samples from 25 cows in herd $A$ each week for 6 weeks were phage typed; the results for 3 cows are illustrated in Table 2 . The number of different phage types present amongst the 4 colonies obtained from any single faecal specimen varied from 1 to 4 , the average being 1.7. The total

Table 2. The phage types of Escherichia coli present in the faeces of 3 cows in herd $A$ at 6 consecutive weekly examinations

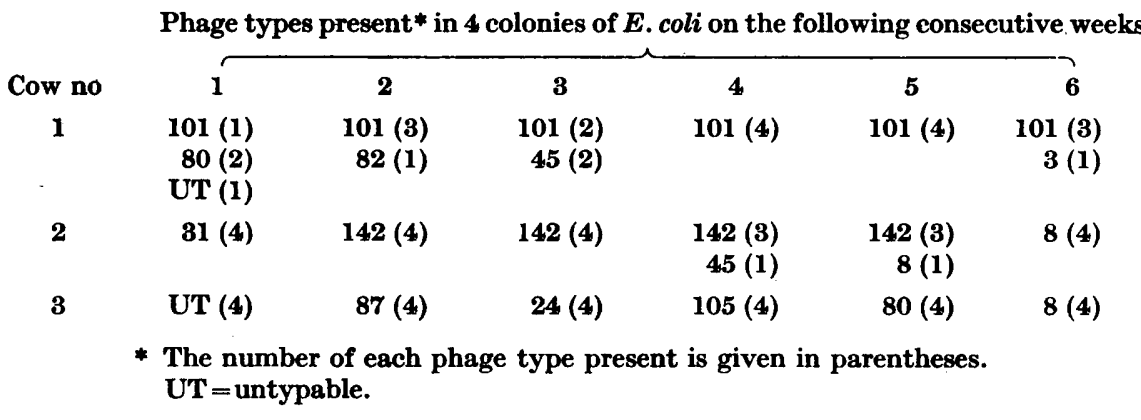

number of phage types found in the 600 cultures examined was $51,65 \%$ belonging to one or other of 10 types; 13 types were found only once. The most common type found was type 101 , forming $16 \%$ of the total cultures examined. The number of different phage types found in each cow over the 6-week period varied from 3 to 10 , the average being 6.2. In some cows the same phage type was present in the faeces at most of the weekly examinations (cow no. 1). Other types were also present but they did not usually persist from week to week (cow no. 2). In other cows one phage-type only was found at several weekly examinations, this type being succeeded in predominance by another type (cow no. 2). A more unusual result was to find a different phage type predominating at every examination (cow no. 3). Despite the fact that the cows were kept in very close proximity to each other and ate the same food there was no evidence to indicate that changes in the Escherichia coli population of any cow from week to week was accompanied by similar changes in any of the other cows. 
The relationship between the phage types of Escherichia coli found in cows and in their calves

The results of typing 10 colonies of Escherichia coli from the faeces of 24 cows and 10 colonies from their calves, both series being obtained when the calves were 4 days old, is shown in Table 3 . All the calves spent the first day of life with their mother. The calves of pairs 1-10 were then given their mother's milk by bucket for the next 4 days; the calves of pairs 11-24 suckled their mothers for these four days, the mother being brought to the calf pen twice daily for this purpose. Even when untypable strains were considered identical,

Table 3. The relationship between the types of Escherichia coli in the faeces of mothers and their calves at 4 days old

\begin{tabular}{|c|c|c|c|}
\hline \multirow[b]{2}{*}{ Pair no. } & \multicolumn{2}{|c|}{ No. of types present in } & \multirow{2}{*}{$\begin{array}{l}\text { No. of } \\
\text { types common } \\
\text { to calf } \\
\text { and mother }\end{array}$} \\
\hline & Calf & Mother & \\
\hline 1 & $\mathbf{1}$ & $\mathbf{2}$ & $\mathbf{0}$ \\
\hline 2 & 4 & 2 & 2 \\
\hline $\mathbf{3}$ & 2 & $\mathbf{5}$ & $\mathbf{0}$ \\
\hline 4 & 2 & 4 & $\mathbf{0}$ \\
\hline 5 & 4 & 2 & 1 \\
\hline 6 & $\mathbf{1}$ & 1 & $\mathbf{0}$ \\
\hline 7 & 1 & 2 & 1 \\
\hline 8 & $\mathbf{3}$ & 5 & 2 \\
\hline $\mathbf{9}$ & $\mathbf{3}$ & $\mathbf{2}$ & $\mathbf{0}$ \\
\hline 10 & 1 & 2 & $\mathbf{0}$ \\
\hline 11 & $\mathbf{3}$ & $\mathbf{3}$ & 2 \\
\hline 12 & 1 & 1 & 1 \\
\hline 13 & $\mathbf{2}$ & $\mathbf{5}$ & 2 \\
\hline 14 & 3 & 1 & 0 \\
\hline 15 & 2 & 1 & $\mathbf{0}$ \\
\hline 16 & $\mathbf{2}$ & $\mathbf{5}$ & $\mathbf{0}$ \\
\hline 17 & 4 & 2 & 0 \\
\hline 18 & 1 & $\mathbf{3}$ & $\mathbf{0}$ \\
\hline 19 & 3 & 2 & 0 \\
\hline 20 & 5 & 2 & 1 \\
\hline 21 & 1 & $\mathbf{2}$ & 1 \\
\hline 22 & 4 & 4 & 1 \\
\hline 23 & 2 & 1 & 0 \\
\hline 24 & 1 & 2 & 0 \\
\hline
\end{tabular}

Ten colonies were examined from each faecal specimen

evidence of some of the phage types of $E$. coli in the faeces of the calf being also present in the faeces of its mother was only found in 10 of the 24 cases; in 6 cases, one type was common to calf and mother and, in a further 4 cases, two types. In pair no. 12 all the colonies examined from mother and calf belonged to phage type 2 indicating that, at least, in this pair the calf had probably acquired its $E$. coli population from its mother. It should be noted in these comparisons that from 1 to 5 types were found at one examination in both cows and calves, the averages being 2.3 and 2.1 respectively, a fact which emphasizes the very limited spread of $E$. coli from mother to off-spring that was occurring. 
In a further experiment, 10 colonies of Escherichia coli isolated at each of 8 weekly examinations from the faeces of each of 6 calves and their 2 foster mothers were phage typed. The calves were kept as 2 groups of three in separate pens, A and B, to which the foster mothers were brought twice daily for suckling. Each group always suckled the same foster mother. At only 3 of the 8 weekly examinations was a phage type found in one or more of the calves in pen $\mathrm{A}$ that was also found at the same time in the foster mother. The corresponding figure for the calves in pen $\mathbf{B}$ and their foster mother was 2. Eleven different phage types were found in all in the faeces of the foster mother of calves in pen A, 5 of which were also found at one time or another in the faeces of her calves. It is significant that 7 of these 11 types were also found in the faeces of calves of pen $\mathrm{B}$, indicating very strongly that the foster mother was probably not the most likely source of the $\boldsymbol{E}$. coli population of her calves. Eight phage types were found in the faeces of the foster mother of the calves in pen $\mathbf{B}$, of which 4 were also found in the faeces of her calves, not one of them being found on more than two occasions. The phage types found most frequently in the calves of pen $B$ were never found in their foster mother. The phage types present in the calves in any pen were much more closely related to each other than to those of the foster mother. Phage type 22 formed $33 \%$ of the cultures examined from the calves in pen B. It was not discovered in either of the foster mothers or in the calves in pen $\mathbf{A}$.

The phage types of Escherichia coli present in the faeces of 8 healthy calves in herd $A$ at weekly examinations during the first 9 weeks of life.

Ten colonies of Escherichia coli, isolated each week for 9 weeks from the faeces of two groups of 4 calves kept in separate pens, C and D, and fed the same bulk milk, were phage typed. Of the total of 72 faecal specimens examined, 20 yielded colonies all of a similar type, 27 of 2 types, 12 of 3 types, 8 of 4 types and 5 of 5 types, the average being $2 \cdot 3$. During the 9 -week-period, 8 to 16 different types were found at one time or another in the faeces of each calf, the average being $12 \cdot 5$. Of the total of 90 cultures examined from each calf the most common type in each individual comprised 17-55 of the cultures, the average being 25 . The number of different phage types discovered in the 720cultures examined from all the calves was 37, 24 being present in the calves of pen $\mathrm{C}$ and 29 in pen $\mathrm{D}$. The 16 types that were common to both pens comprised $94.5 \%$ of the total number of cultures examined from the calves in pen $\mathrm{C}$ and $85 \%$ of those from pen $\mathrm{D}$.

As in the case of the cows examined at weekly intervals, considerable differences were noted between individual calves. Two calves in pen $\mathbf{C}$, for example, yielded cultures predominantly consisting of phage type 2 for 3 weeks, this type being succeeded by other types for 3 weeks, and then being replaced in predominance by phage type 2 again in the final 2-3 weeks. By contrast, the faeces of another calf in this pen yielded a number of types, not one of which was predominant on more than 1 week.

Comparing the results for pens $\mathrm{C}$ and $\mathrm{D}$, some types were more commonly found in one group than in the other. For example, 98 cultures from the 
calves in pen $\mathrm{C}$ belonged to phage type 2 , and 8 to phage type 22 ; the corresponding figures for pen $D$ were 16 and 56, respectively. When the results of each weekly examination were compared, it was noted that the phage types present in the calves in the same pen generally bore more relationship to each other than they did to those from the calves in the other pen. However, the Escherichia coli present in the faeces of some individual calves bore no relationship at all to those of the other calves examined at the same time. Some phage types made a relatively sudden appearance in the faeces of a number of calves in the same pen at the same time and continued to be found for varying periods afterwards. For example, phage type 37 was the predominant phage type in the calves of pen $\mathrm{C}$ at the fourth weekly examination; it had never been discovered in them at the previous examinations and was not found in the calves in pen D. Again, phage type 22 suddenly appeared in 3 of the 4 calves in pen $\mathbf{D}$ on the seventh week and was found commonly in them at subsequent examinations. It was also found in one of the pen $\mathrm{C}$ calves in the seventh week but not subsequently.

\section{The phage typing of Escherichia coli isolated from the faeces of healthy and scouring calves at daily examinations}

The results of phage typing 10 cultures of Escherichia coli obtained every day for 12 days from the faeces of 13 calves, 8 of which developed white scours during this period, is illustrated in Table 4. The first examination was performed at the end of the first day after birth. A further 7 cases in which examinations had not been made before scouring were also studied; the results were similar to those shown in Table 4. Unless stated, no calf was treated with streptomycin. The number of different phage types in the faeces of the healthy and scouring calves at each examination of 10 colonies varied from 1 to 5 . The average was 2.0 for the healthy calves, one type only being found in $38 \%$ of the examinations. The corresponding figures for scouring calves was $2 \cdot 1$ and $37 \%$ during the scouring period and $2 \cdot 4$ and $22 \%$ during the non-scouring period. No one phage type was found to persist in the faeces of any of the healthy calves throughout the 12 daily examinations, although type 71 was found in the faeces of calf 3 on all except the fifth day, being the major type present on 4 days. The general finding was that one type predominated for a few days, and then was gradually succeeded by another type which might in turn be succeeded by another, although on many days, 3, 4 or even 5 different types were found amongst the 10 colonies examined. The number of different phage types found in the faeces of each calf during the 12 days varied from 3 to 13 , the average being 8 .

The picture was essentially the same in scouring calves as in healthy calves, this being particularly noticeable in calf 13 in which the scouring period was long. In this calf, phage type 96 was predominant in the early scouring period, being gradually superseded in predominance by phage type 2, and, then by phage type 71 which was finally gradually replaced by phage type 80 ' the disease process then being arrested by the oral administration of streptomycin. In many cases, the phage type most commonly found during the 


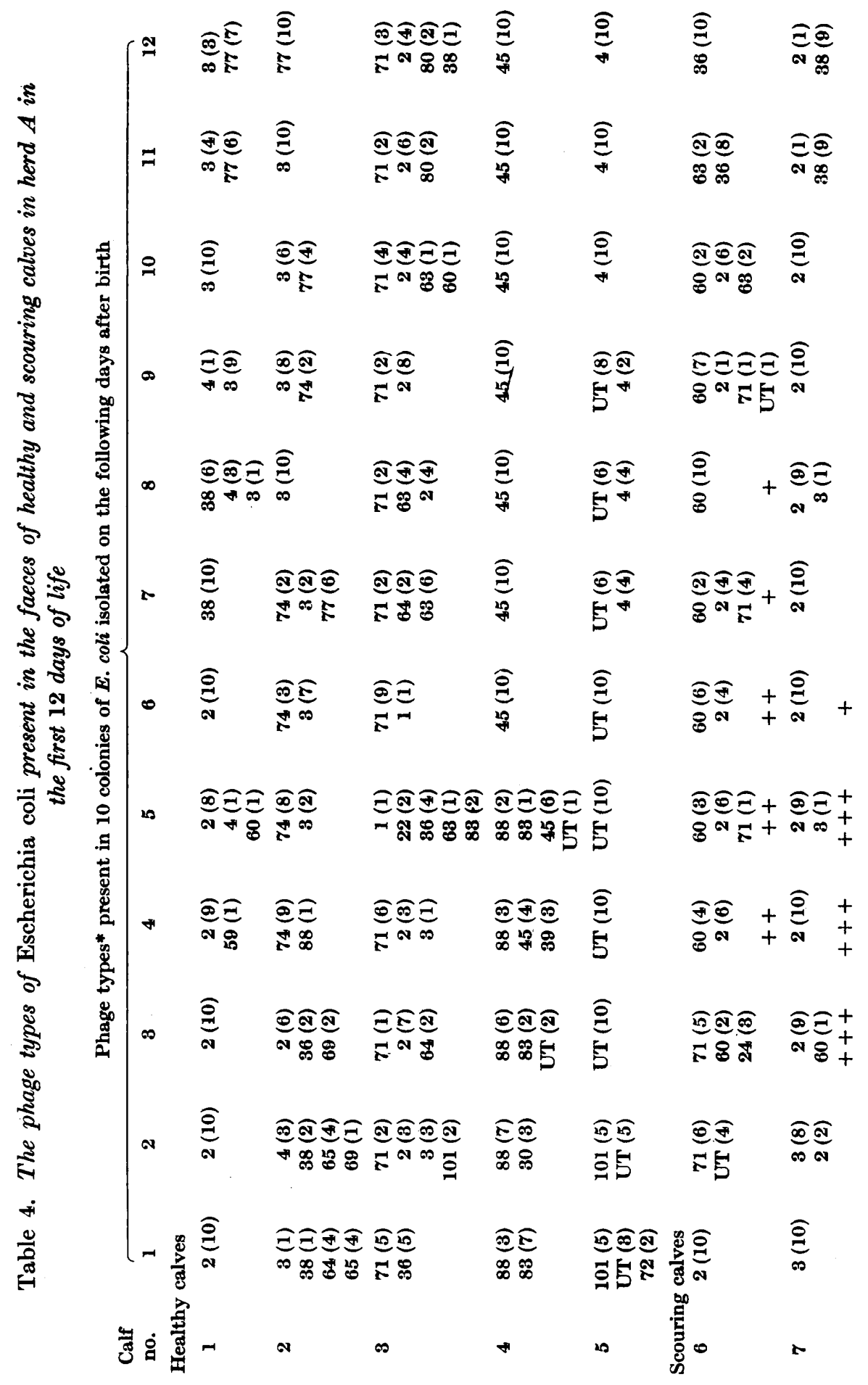




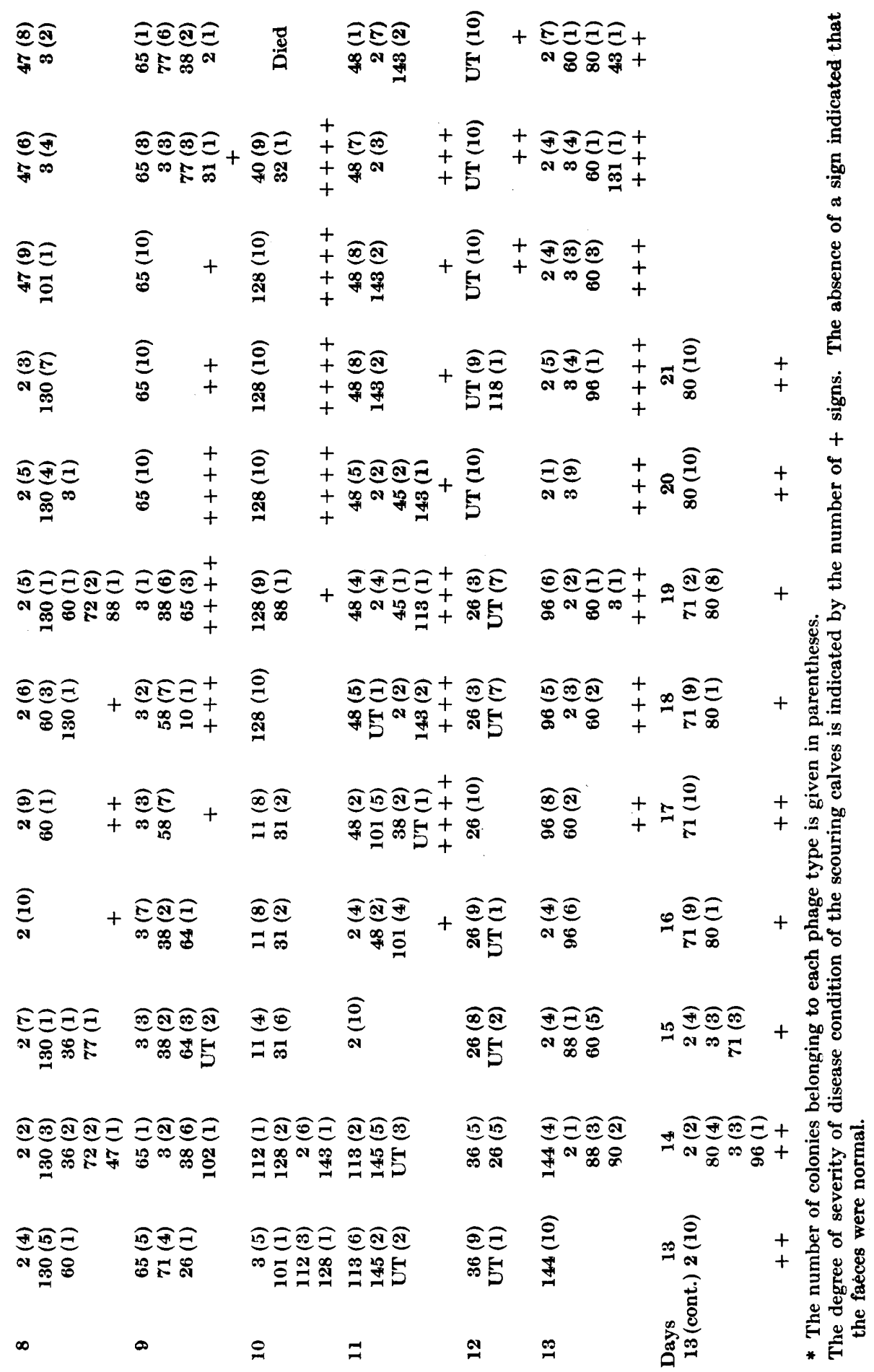


scouring period was present in the faeces for a number of days before scouring began and invariably afterwards. Sometimes there was an interval in the prescouring period in which that phage type was not found in the faeces. For example, phage type 2 was the only type discovered in the faeces of calf 6 at the end of the first day after birth; it was not present at the second and third day but reappeared when scouring commenced on the fourth day, and was not found at all on the final day of scouring. Further examples of this are seen in the case of phage type 65 and calf 9 and phage type 128 and calf 10 . During the scouring period, 34 types were found in all in the 15 scouring calves examined, 12 of the types occurring reasonably frequently. The number of different phage types in each calf during the scouring period varied from 1 to 8 , the average being 4 . No one particular phage type was found to be present in more than 6 of the 15 cases and in only 4 of these was it present in considerable numbers. This type (phage type 2) was also found in the faeces of 3 of the 5 healthy calves in this experiment, being present in considerable numbers in 2 of them. In all observations (Table 1) it was discovered to be the most frequent type found in healthy and scouring calves in herd A. The next most common type in the present experiment was phage type 71 which was found in 5 scouring calves, being present in considerable number in 3 of them. It is noteworthy that phage type 71 was also found in healthy calf 3 at all except one of the twelve examinations. Phage types 71 and 2 were very commonly found in the faeces of calves in the first half of the calving season, when most of the calves were born and when the incidence of white scours was low.

\section{The possibility of bacteriophages influencing the distribution of different phage types in the intestinal contents of calves}

It was foreseen that qualitative changes in the Escherichia coli population of the faeces of scouring and healthy calves might be brought about by the selective action of bacteriophages present in the intestinal tract. Consequently, many of the faecal specimens examined in the previous experiment were retained in the refrigerator until the phage typing results were available. When a change in predominating type occurred in the faeces of a calf the faeces collected from this calf on the previous day and on the same day as the change occurred were examined for the presence of phage that was lytic for the phage type of Escherichia coli previously predominant. For example, in the case of calf no. 1 (Table 4), the faeces collected on the sixth and seventh days were examined for phages active on phage type 2 . Over forty examinations of this kind were conducted; only in two were positive results obtained, the amount of phage present in those two being so small as to make very remote the possibility that phage had significantly influenced the number of the E. coli on which they were active. A more remote possibility, at least in shortterm studies, was that change of phage type might be induced by phage in the intestinal tract bringing about lysis of the predominant strain with the emergence of phage-resistant variants of the predominating strain, as distinct from permitting other strains to occupy the dominant position. If such variants influenced the typing results obtained with the routine test phages, they would 
be acted upon by fewer of the test phages that acted upon the strain from which they were derived, and not at all by the other test phages. A comparison of the phage reactions of the coli types found in the same calf at successive examinations yielded no positive evidence of this having occurred.

\section{The number of Escherichia coli present in the faeces of healthy and scouring calves in herd $A$}

The approximate numbers of viable Escherichia coli present in the faeces of 8 healthy calves at different times after birth is illustrated in Table 5. Very high counts (c. $10^{8}$ bacteria/g. faeces) were observed at 2 days after birth. Counts remained high for 14 days, after which they decreased until at 50 days they were quite low. Very low counts were also observed at 150 days after birth.

Table 5. The number of Escherichia coli present in the faeces of healthy calves at different times after birth

No. $\times 10^{4}$ of viable $E$. coli/g. of faeces at the following days after birth

\begin{tabular}{|c|c|c|c|c|c|c|}
\hline Calf no. & 2 & 4 & 7 & 14 & 50 & 150 \\
\hline 21 & 120,000 & 12,000 & 20,000 & 6,000 & 1 & 10 \\
\hline 22 & 250,000 & 10,000 & 100,000 & 6,000 & 150 & 2 \\
\hline 23 & 50,000 & 2,000 & 40,000 & 30,000 & 5 & 30 \\
\hline 24 & 200,000 & 7,000 & 60,000 & 50,000 & $\mathbf{3}$ & 500 \\
\hline 25 & 200,000 & 7,000 & 60,000 & 50,000 & 3 & 50 \\
\hline 26 & 600,000 & 40,000 & 50,000 & 10,000 & 3 & 10 \\
\hline 27 & 50,000 & 10,000 & 25,000 & 600 & 1500 & 100 \\
\hline 28 & 40,000 & 1,200 & 5,000 & 3,000 & 10 & 50 \\
\hline
\end{tabular}

As compared with faeces from young healthy calves, low counts of Escherichia coli were obtained from the faeces of cows, e.g. the approximate numbers in the faeces of 10 cows were $80,50,30,25,5,5,5,3,1$ and $0.3 \times 10^{4} / g$, respectively.

The numbers of Escherichia coli present in the faeces of scouring calves were similar to those for the healthy calves shown in Table 5 . The numbers present, in calves scouring at 4 days of age were $7,500,9,000,120,00036,000 \times 10^{4} / \mathrm{g}$. faeces, and in calves scouring at 7 days of age were $60,000,40,000,200,000$ and $25,000 \times 10^{4} / \mathrm{g}$. faeces. Counts carried out on the faeces of calves that developed white scours were also made before, during and immediately after the scouring period. There was no significant difference between the results for each of these three periods when each calf was considered individually.

The phage types of Escherichia coli in the faeces of calves during outbreaks of white scours in different herds

The distribution of different phage types among 5 colonies of Escherichia coli isolated from the faeces of scouring calves during outbreaks of white scours in $\mathbf{2 7}$ herds other than herd A was also studied. All the calves from each herd were scouring at the same time, the time when the faecal samples were examined. It was also possible to examine faecal samples from healthy calves 
in herds where scouring calves were present. The history of these herds was much less complete than that of herd A. Most of the herds were not selfcontained, some calves being purchased in the market.

The number of phage types present amongst 5 colonies of Escherichia coli isolated at each examination of the faeces of healthy and scouring calves varied from 1 to 5 . The average for faecal specimens from 61 scouring calves at each examination was $1 \cdot 7$, one phage type only being found in the faeces of 28 calves. The corresponding figures for 45 healthy calves was 1.9 and 16 respectively.

Although one or two phage types of Escherichia coli were found more frequently in one herd than in another, there was no evidence to indicate that one type only was associated with white scours in any particular herd at any particular time. It is noteworthy that in two herds different phage types were found in the faeces of each calf. As in the case of herd A, the phage types present in the scouring calves were also found not infrequent in the faeces of healthy in-contact calves.

\section{DISCUSSION}

The fact that many more phage types of Escherichia coli were identified in herd $A$ alone by means of phage typing than has been found by other workers who have approached the study of $E$. coli infection in calves by serological methods is not surprising since Nicolle et al. (1952), working with strains of $E$. coli of human origin, found that several different phage types could be found amongst strains of the same serological type. A possible explanation of this is that many of the phage types differ from each other by means of acquired phage resistance only, i.e. some strains through contact with different phages in the field have become resistant to them and as a result their susceptibility to the test phages may have altered; such strains would then accordingly be differentiated from otherwise identical strains which had been exposed to other and different phages. Positive evidence exists that such changes take place in other species of bacteria and is responsible for some type differentiation (Smith, 1948; $1951 a, b$; Felix \& Anderson, 1951; Anderson $\&$ Felix, 1953; Tee, 1955). Since faeces are the natural habitat of $E$. coli and are also a good source of phages active against these organisms it is not difficult to imagine these phages having an impact on phage type differentiation. Such changes would probably occur more infrequently and more slowly than in vitro experiments indicate and, consequently, they would not be a source of confusion in short-term studies. This method of typing probably classified together strains with a similar history of phage infection and at the same time may differentiate from each other strains essentially the same except for a dissimilar history of phage infection. Its use, therefore, is probably restricted to short-term epidemiological investigations.

Up to eight phage types were found during the examination of 150 colonies of Escherichia coli obtained from the same specimen of faeces, some of the types only occurring once or twice. This, considered with the fact that each gram of calf faeces may contain $10^{\circ} \mathrm{E}$. coli indicates the magnitude of the task 
of studying the qualitative aspects of the $E$. coli population of the alimentary tract. It is obviously only possible by present methods to obtain information on the dominant type or types. Many more types may be present in quite considerable numbers, their presence being masked by the overwhelming numbers of the dominant types. However, the present studies have thrown some light on the behaviour of $E$. coli in the calf alimentary tract. It is apparent that the alimentary tract of each calf has to be considered as a separate entity since the $\boldsymbol{E}$. coli flora of some individual calves occasionally bore no resemblance to that of other calves kept in the same pen and given the same milk for as long as 9 weeks. In general, however, a much greater degree of similarity existed between the flora of calves kept in the same pen than between calves kept in different pens but given the same bulk milk. The phage typing results on such groups of calves tends to indicate that the pens themselves may be the most likely source of origin of the $\boldsymbol{E}$. coli found in the calves. In view of the almost constant habit of calves of sucking animate and inanimate objects it is not difficult to visualize this possibility. The mother did not appear to be a potent source of origin of the $E$. coli population of the alimentary tract of her calf. It is conceivable, though, that the phage types found in some of the calves may also have been present in the faeces of the mother in undetectable numbers.

The change of the predominating phage types which occur in the intestinal tract is interesting. The same phage type remained dominant in some calves and cows for many weeks and frequent and relatively sudden changes took place in others; the usual picture was of a gradual change of predominating type during 1-2 weeks, thus resembling in many respects what happens in man (Sears, Brownlee \& Uchiyama, 1949; Sears \& Brownlee, 1951).

Qualitatively and quantitatively, the behaviour of Escherichia coli in the alimentary tract of scouring calves so closely resembled that of the healthy calves that one might wonder what part the $E$. coli played in the disease process of the scouring calves. The association of these bacteria with white scours has, however, now been well established (see Lovell, 1955). Fresh evidence was obtained from chemotherapeutic studies on many of the scouring calves in the present investigation. For example, ever since herd $\mathbf{A}$ had been established, the oral administration of streptomycin had had a very significant effect in controlling white scours. One dose usually resulted in a complete sessation of diarrhoea, the faeces being of normal consistency on the following and succeeding days and the number of $E$. coli present being almost negligible. Only one treated calf died during the present investigation, this calf having been left too long before being treated. The evidence from the other herds is even more convincing (Smith \& Crabb, 1956). In these the $E$. coli in the faeces of scouring calves before and after treatment were tested for sensitivity to chemotherapeutic agents. When they were sensitive to streptomycin, therapy with this agent was almost invariably successful, but when they were resistant, it was of no avail; treatment with other agents to which the $\boldsymbol{E}$. coli in the faeces were sensitive yielded satisfactory results. There can be little doubt, therefore, that $E$. coli played an important role in the cases of white scours studied in the present investigation. Considering all the evidence it is apparent that less 
emphasis should be placed on regarding white scours as a disease associated with a relatively few specific types of $E$. coli. It would be advisable to regard the development of the disease in some calves in a herd as an adverse result of the endeavour of the calf to establish an equilibrium with the types of $E$. coli that have colonized its alimentary tract as suggested by Lovell (1955), the present studies stressing that the actual phage types of $E$. coli present in the alimentary tract are of less importance than the resistance of the calf. This view does not preclude the possibility that there may be herds in which specific strains are mainly responsible for the scours. It merely indicates that scours may still occur in these herds in the absence of the specific strains.

We are grateful to Professor R. Lovell for his help in planning these studies and in preparing this paper for publication. Our thanks are also due to Messrs Beech, Honey and Johnson for their assistance at various stages of the work.

\section{REFERENCES}

Anderson, E. S. \& Feurx, A. (1953). The Vi-type determining phages carried by Salmonella typhi. J. gen. Microbiol. 9, 65.

Felix, A. \& Anderson, E. S. (1951). Bacteriophages carried by the Vi-phage types of Salmonella typhi. Nature, Lond. 167, 603.

Fisk, R. T. (1942). Studies on staphylococci. I. Occurrence of bacteriophage carriers amongst strains of Staphylococcus aureus. II. Identification of Staphylococcus aureus strains by means of bacteriophage. J. infect. Dis. 71, 153, 161.

FredericQ, P. (1950). Examination of colicine-producing strains for lysogenicity. C.R. Soc. Biol., Paris, 144, 728.

Lovell, R. (1955). Intestinal diseases of young calves with special reference to infection with Bacterium coli. Vet. Rev. Ann. $1,1$.

Nicholle, P., Le Minor, L., Buttiaux, R. \& Ducrest, P. (1952). Phage-typing of Bact. coli strains isolated in infantile gastro-enteritis. 1. Schema of types characterized. Bull. Acad. nat. Med. 136, 480.

Sears, H. J. \& Brownlee, I. (1951). Further observations on the persistence of individual strains of Escherichia coli in the intestinal tract of man. J. Bact. 63, 47.

Sears, H. J., Brownlee, I. \& Uchiyama, J. K. (1949). Persistence of individual strains of Escherichia coli in the intestinal tract of man. J. Bact. 59, 293.

Sмітн, H. W. (1948). Investigations on the typing of staphylococci by means of bacteriophage II. The significance of lysogenic strains in staphylococcal type designation. J. Hyg., Camb. 46, 82.

Sмгтн, H. W. (1951 a). The typing of Salmonella thompson by means of bacteriophage. J. gen. Microbiol. 5, 472 .

Sмгтн, H. W. (1951 $b$ ). The typing of Salmonella dublin by means of bacteriophage. J. gen. Microbiol. 5, 919.

Smith, H. W. \& CrabB, W. E. (1956). The sensitivity to chemotherapeutic agents of a further series of strains of Bacterium coli from cases of white scours: the relationship between sensitivity test and response to treatment. Vet. Rec. 68, 274.

TEE, G. H. (1955). Bacteriophage typing of Shigella sonnei and its limitations in epidemiological investigation. J. Hyg., Camb. 53, 54.

Wilson, G. S. \& Atkinson, J. D. (1945). Typing of staphylococci by the bacteriophage method. Lancet, i, 647 .

Woon, P. C. (1955). The epidemiology of white scours among calves kept under experimental conditions. J. Path. Bact. 70, 179.

(Received 18 May 1956) 